There was no significant correlation between the number of wheezing attacks ( $p>0.05)$.

No correlation between vitamin $\mathrm{D}$ levels and acute bronchiolitis was found but this may be the result of few numbers of patients that we studied with.

\section{EVALUATION OF IL-6 AND HIGHLY SENSITIVE CRP VALUE IN CSF AND SERUM CHILDREN REFERRED TO PEDIATRIC EMERGENCY ROOM}

doi:10.1136/archdischild-2012-302724.0829

${ }^{1} \mathrm{~A}$ Hamedi, ${ }^{2} \mathrm{H}$ Ayatollahi. 'Pediatrics; ${ }^{2}$ Mashhad University of Medical Sciences, Mashhad, Iran

Introduction Acute bacterial meningitis which is a pediatric emergency with high mortality and morbidity, must be diagnosed and treat promptly. Determination of some inflammatory mediators example IL- 6 and hsCRP were useful in differential diagnosis of bacterial and viral meningitis.

Objectives Determination of hs CRP and IL6 in serum and CSF in children suspected meningitis.

Materials and Methods From of children that hospitalized in pediatric emergency ward and for they perfomed lumbar puncture, $1 \mathrm{cc}$ serum and csf of they were taken to libratory ward and have measured IL-6 with Elisa method and hsCRP with Immunoturbidometry method patients were fallowed up and Finally we compared levels of this two mediators.

Results From 81 cases, 27 cases $(33 / 3 \%)$ were bacterial meningitis 27 cases $(33 / 3 \%)$ were viral meningitis and 27 cases (33/3\%) were Normal. IL- 6 concentration in the csf and serum were significantly raised in cases of bacterial meningitis $(\mathrm{P}=0 / 001, \mathrm{P}=0 / 01)$ but hsCRP concentration in the CSF and serum we not significantly raised in cases of bacterial meningitis ( $p=0.46, p=0.29$ ). Mean IL- 6 in serum in bacterial meningitis was (50.01) and in viral meningitis was (10.64) mean IL-6 concentration in the CSF in bacterial meningitis was (180.74) and in viral meningitis was (39.08) mean hsCRP in CSF in bacterial meningitis was (2.22) and viral meningitis was (1.29).

Conclusion The measurement of IL- 6 in the CSF and serum is potentially a very useful diagnostic tool for differential diagnosis of bacterial and viral meningitis.

\section{THE VALUE OF MEAN PLATELET VOLUME IN THE DETERMINATION OF COMMUNITY ACQUIRED PNEUMONIA IN CHILDREN}

doi:10.1136/archdischild-2012-302724.0830

'E Karadag Oncel, 'Y Ozsurekci, 'A Kara, ${ }^{2} S$ Karahan, 'AB Cengiz, 'M Ceyhan. ${ }^{1}$ Pediatric Infectious Disease, Hacettepe University Ihsan Dogramaci Childrens' Hospital; ${ }^{2}$ Biostatistics, Hacettepe University Faculty of Medicine, Ankara, Turkey

Background The aim of this study was to evaluate whether mean platelet volume (MPV) could be used for the diagnostic tool of community acquired pneumonia (CAP) and for making the decision for hospitalization.

Methods The computerized records of children aged 1 to 18 years who were diagnosed with CAP based on WHO criteria were evaluated. A standard protocol was followed, and patients with severe CAP were hospitalized. CAP patients were divided into two groups based on disease severity. The control group consisted of age- and gender-matched healthy children who attended the "Well-child" clinic during the study period. Values for hemoglobin, white blood cell count (WBC), platelet count, MPV and $\mathrm{C}$-reactive protein (CRP) obtained on first presentation were recorded for each patient.

Results A total of 139 patients were diagnosed with CAP during the study period, 82 (58.9\%) of which had severe disease, which required hospitalization, while the remaining 57 (41.4\%) were followed-up as outpatients. The control group consisted of 61 healthy children Patients with CAP had lower MPV values than their healthy counterparts (7.14 \pm 0.70 vs. 8.35 $\pm 1.26 \mathrm{fL}$; $\mathrm{p}<0.001)$. Following ROC analysis, the cut-off value established for MPV was 8.05, which had a sensitivity of $91 \%$ and a specificity of $51 \%$ for making a diagnosis of CAP. Patients with severe CAP that required hospitalization had significantly higher CRP levels than those who were followed-up on an outpatient basis $(\mathrm{p}<0.001)$.

Conclusions Our findings suggest that MPV could be used for the diagnosis of CAP in children.

\section{AN ANALYSIS OF THE PROGRESSIVE CLINICAL DETERIORATION IN THE CASES WITH SUBACUTE SCLEROSING PANENCEPHALITIS}

doi:10.1136/archdischild-2012-302724.0831

S Saygili, C Yarar, G Arslan, E Aksuyek. Pediatrics, Turkish Ministry of Health, Kanuni Sultan Suleyman Training and Research Hospital, Istanbul, Turkey

Subacute sclerosing panencephalitis (SSPE) is devastating progressive neurodegenerative disorder of the central nervous system by an aberrant measles virus. SSPE is still important health problem in countries where measles immunization is incomplete and in world regions where genetic polymorphism to this particular infection is present. We aimed to make a retrospective analysis of the clinical and laboratory findings and prognostic factors of children with SSPE.

Method A total 55 consecutive cases (22 female, 33 male), aged 5-19 years, followed-up for SSPE at the Pediatric Neurology Department were included. The risk factors for progression time of SSPE from stage I-II to IV according to the criteria of Jabbour examined were gender, age at diagnosis of SSPE, presence of neuroimaging abnormalities, age of patient during measles infection and the antibody index for measles. SSPE risk factors were obtained through a questionnaire via personal interviews and the medical records and were assessed using Mann-Whitney U, Student $t$ and Pearson correlation tests.

Results Presence of neuroimaging abnormalities, gender, age at diagnosis of SSPE, presence of neuroimaging abnormalities, age of patient during measles infection and the antibody index for measles were not associated with a progressive clinical deterioration ( $p>0.05) .66 .7$ percent of cases were vaccinated with measles vaccine, 43.5 percent of cases have had measles infection before age of 9 months.

Conclusion Viral, host and environmental factors are probably involved in the development and progression of SSPE. Successful measles vaccination programmes which accordance with the requirements of that country can protect the population against SSPE.

\section{ACUTE TRANSVERSE MYELITIS IN A PEDIATRIC CASE OF LYME DISEASE}

doi:10.1136/archdischild-2012-302724.0832

'i Erol, ${ }^{2 B}$ Kiliçaslan, ${ }^{3}$ S Demir, ${ }^{4} \mathrm{H}$ Gülcan, ${ }^{1} \mathrm{~F}$ Alehan. 'Department of Pediatrics, Division of Child Neurology; ${ }^{2}$ Department of Pediatrics; ${ }^{3}$ Department of Radiology; ${ }^{4}$ Department of Pediatrics, Division of Neonatalogy, Baskent University Hospital, Adana, Turkey

Background and Aim A broad range of neurologic disorders have been described in children with Lyme disease. In contrast, there are few reports of transverse myelitis in pediatric Lyme disease patients. Case Report A 12-old girl admitted with the complaints of weakness in legs, sensory loss in lower limb, and backache for 10 days. Mental status, cranial nerve, and upper-extremity motor and sensory examinations produced normal results. The strength in his iliopsoas and hamstring muscles was scored 2/5. Deep tendon reflexes 
were absent in both lower extremities. Babinski's signs were not noted. The Romberg sign was present. Magnetic resonans imaging (MRI) of the spinal cord revealed enhancing $T_{2}$ hyperintensity within the spinal cord, from $T_{10}-L_{1}$. (Figure 1). Cerebrospinal fluid (CSF) showed no pleocytosis and normal protein and glucose concentrations. The CSF did not show oligoclonal banding and immunglobulin (Ig)G index was normal. Serum serologic evaluation of Borrelia burgdorferi was positive for IgM but negative for IgG. CSF serologic evaluation was negative (both ELISA and Western blot). The patient was diagnosed as manifesting acute transverse myelitis. He was treated with intravenous methylprednisolone pulse therapy (1000 mg/day for 5 consecutive days), followed by oral prednisolone (1 mg/kg per day). After the start of steroid therapy, the patient showed gradual clinical improvement and was able to walk on the 30 th day of illness. We also administered doxycycline $4 \mathrm{mg} / \mathrm{kg}$ per day for 14-days for borreliosis.

Conclusion This case serves as a reminder that acute transverse myelitis can be a rare clinical manifestation of Lyme disease.

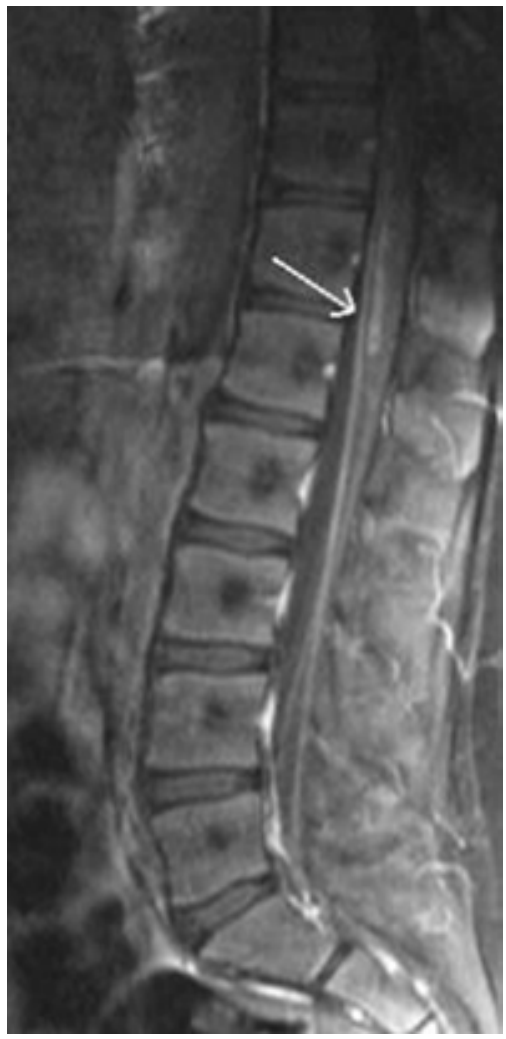

Abstract 832 Figure 1

\section{THE EFFECT OF PROBIOTICS ON COLONIZATION OF RESISTANT ORGANISMS IN PRETERM INFANTS}

doi:10.1136/archdischild-2012-302724.0833

${ }^{1} A$ Kurt, ${ }^{1} A$ Ecevit, $' D$ Anuk Ince, ${ }^{1} A$ Abbasoglu, ${ }^{2} 0$ Kurt Azap, 'Z Ecevit, ${ }^{3} E$ Ogus, 'A Tarcan. 'Pediatrics; 'Infectious Diseases; ${ }^{3}$ Biostatistics, Baskent University Faculty of Medicine, Ankara, Turkey

Background and Aims To investigate the effect of probiotics on colonization of resistant organisms in preterm infants receiving antibiotics.

Methods This study comprised of preterm infants who were born $<36$ weeks and received antibiotic treatment or prophylaxis. Preterm infants were divided into two groups according to receiving probiotic (Lactobacillus reuteri). Stool culture and nasal swab culture were taken to determine colonization.
Results In the group receiving probiotics, mostly colonizing the stool cultures bacteries were Klebsiella spp, Escherichia coli, Enterococcus spp., Enterobacteriae spp., Staphylococcus spp. respectively, and in the group not receiving probiotic mostly colonizing the stool cultures bacteries were Klebsiella spp, Enterococcus spp., Staphylococcus spp., Escherichia coli, Enterobacteriae spp. respectively. When probiotic receiving group compared was with not receiving group, proliferation rate of stool cultures was higher in probiotic group. In the groups receiving and not receiving probiotic, proliferation of the nose cultures were similar. Increase in the proliferation rates of weekly stool cultures in probiotic receiving group was statistically significant but there was no statistically difference in the proliferation rates of nose and other cultures that were taken weekly. There was no statistical difference in both groups in the development of resistant organisms.

Conclusions The use of probiotics in neonatal intensive care unit for premature infants who received treatment with antibiotics, did not prevent the colonization of pathogenic microorganisms.

\section{THE SENSITIVITY OF BACTERIAL MENINGITIS SCORE IN CHILDREN WITH ACUTE MENINGITIS IN OATAR}

doi:10.1136/archdischild-2012-302724.0834

${ }^{1} \mathrm{H}$ Abdlrahman, ${ }^{1} \mathrm{~A}$ Alhamadi, ${ }^{1} \mathrm{~K}$ Al-Saddi, ${ }^{1} \mathrm{~W}$ Seleem, ${ }^{1} \mathrm{H}$ Badran, ${ }^{1} \mathrm{~N}$ Sharaf, ${ }^{2} \mathrm{~A}$ Farooq. ${ }^{1}$ Hamad Medical Center; ${ }^{2}$ Aspitar Medical Center, Doha, Oatar

Background The empirical use of antibiotics in children with suspected meningitis is a common clinical practice worldwide that often leads to drug resistance. It is difficult to clinically differentiate bacterial when compared to viral meningitis until a culture study ofcerebral spinal fluid (CSF) Or CSF viral PCR study is performed. A 'wait and see' approach may lead to undesirable outcome. Bacterial Meningitis Score (BMS) is a tool that was developed to help physicians to differentiate between viral versus bacterial meningitis.

Aim To determine the usefulness if any of BMS for discriminating between bacterial or viral meningitis is young children.

Methodology We retrospectively reviewed the charts of all children (from birth till 14 years old) who were admitted with the diagnosis of meningitis to Hamad general hospital in last 2 years period. Result A total 120 patients (68\% boys) with confirmed meningitis were reviewed during the study period. The mean age was $(6.3 \pm 2.7$ year). The majority of patients $112(93.3 \%)$ had viral type meningitis while the remaining had bacterial meningitis (Strep Pneumia, Neisseria meningitis and $\mathrm{H}$. Influenza). The sensitivity of BMS tool revealed a sensitivity of $100 \%$ (95\% CI: 75.1 to 100.0$)$ and a specificity of $60.9 \%$ (95\% CI:50.1-69.7).

Conclusion Our study shows that BMS is a simple, easy and highly sensitive tool that can differentiate bacterial from viral meningitis and it is use may limit the use of unnecessary antibiotic s and hospitalizations.

\section{THE VALUE OF A POSITIVE NITRATE TEST IN ROUTINE URINE ANALYSIS FOR THE DIAGNOSIS OF CHILDHOOD UTI}

doi:10.1136/archdischild-2012-302724.0835

HA Bin-Nakhi, E Bu-Hamrah, S Sadeq, AR Moodambail. Pediatrics, Adan Hospital, Kuwait, Kuwait

Urinary tract infection (UTI) is one of the most common diseases in children. Its diagnosis depends mainly on urine culture. Urinalysis is used as a screening test to exclude UTI. A hospital based, prospective case-review study was carried out at the Pediatric Department, Al-Adan Hospital, State of Kuwait. The objectives of this study is:

1. To determine the proportion of positive urine cultures identified in patient less than 12 years of age, admitted to hospital and was found to have a positive nitrite test in the routine urinanalysis. 